\title{
Prostatakarzinomrisiko
}

\section{Gefrorenes frittieren kann fatal sein}

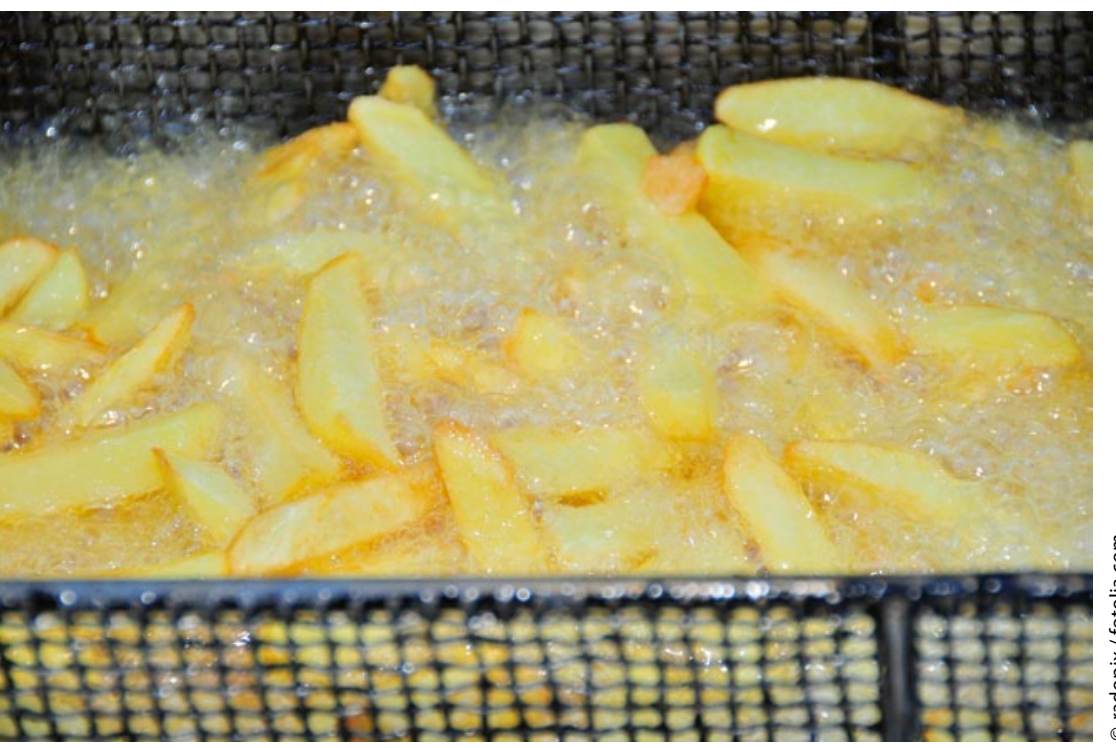

Krebsgefahr aus der Friteuse
— Wer regelmäßig frittierte Tiefkühlkost isst, hat ein höheres Prostatakarzinomrisiko. Diesen Zusammenhang haben USamerikanische Wissenschaftler mit einer Fall-Kontroll-Studie belegt. Anhand von jeweils rund 1.500 Erkrankten und Kontrollpersonen ermittelten die Forscher die Gewohnheiten, sich für Donuts, Pommes und anderes typisches „Fastfood" aus der Kühltruhe zu bedienen. Männer mit hohem Konsum an Gefrorenem hatten ein höheres Karzinomrisiko - zudem mit aggressiveren Formen von Prostatatumoren [Stott-Miller $\mathrm{M}$ et al. Prostate 2013; 73: 960-9]. Das abgefragte Kriterium bestand darin, ob sich die Männer mindestens einmal wöchentlich oder höchstens einmal monatlich von solchen Nahrungsmitteln nährten. Bei Ersteren, den wöchentlichen Konsumenten, lag das Tumorrisiko um etwa $35 \%$ höher. Die Autoren verweisen auf den bekannten Zusammenhang zwischen Frittieren und Karzinogenese: also der Bildung von Acrylamid, heterozyklischen Aminen, Aldehyden und Acrolein. „Diese Substanzen bilden sich vornehmlich, wenn dasselbe Frittierfett wiederholt zum Auftauen und Garen verwendet wird", so die Wissenschaftler.

Martin Roos

\section{Harnblasenkarzinomrisiko}

\section{Frühstücksei bedenklich?}

\section{Eltern und Kinder befragen}

— Um Symptome des unteren Harntrakts (LUTS) im Kindesalter zu erfassen, ist ein Fragebogen oft wenig hilfreich. Probleme beim wahrheitsgemäßen Ausfüllen haben nämlich nicht nur jüngere Kinder, sondern bisweilen auch die Eltern [Selcuk M et al. Urology 2013, online 13. Mai; doi: 10.1016/j.urology.2013.03.021].

Türkische Urologen hatten 272 Kindern und ihren Eltern den speziell für pädiatrische Inkontinenzprobleme entwickelten Selbstauskunftsbogen ICIQ-CLUTS vorgelegt. Der Fragebogen liegt sowohl in einer Kinder- als auch in einer Elternversion vor. Die Kinderversion wurde überwiegend korrekt ausgefüllt. In der Altersgruppe der Fünf- bis Neunjährigen gab es allerdings - wenig überraschend - erhebliche Probleme. Aber auch die Elternversion hatte ihre Tücken. Vor allem Eltern, deren Kinder in der (Vor-)Pubertät steckten, lagen bei den Angaben zu körperlichen und seelischen Problemen ihrer Kinder im Zusammenhang mit der Blasenstörung oft daneben. Eltern von Kindern im Alter zwischen zehn und 13 Jahren schnitten im Erwachsenenfragebogen genauso schlecht ab wie die Fünf- bis Neunjährigen in ihrem Kinderfragebogen. Bei Jugendlichen ab zehn lieferte der Kinderfragebogen dagegen überwiegend zuverlässige Ergebnisse. Der elterliche Eindruck von der Blasenstörung ihres Kindes weicht offenbar oft deutlich von der Wahrnehmung des Patienten ab, folgern die Autoren. Es sei daher in jedem Fall sinnvoll, sowohl die elterliche als auch die kindliche Sicht zu berücksichtigen. Das gelte vor allem dann, wenn die Patienten in die Pubertät kommen.

Dr. Elke Oberhofer
_ In den 1980er-Jahren kam zum ersten Mal der Verdacht auf, der Konsum von Hühnereiern könnte im Zusammenhang mit einem erhöhten Blasenkarzinomrisiko stehen. An die quantitative Auswertung der seit mittlerweile 30 Jahren laufenden Forschung machten sich nun chinesische Urologen. Eigentlich könnten die Ergebnisse ihrer Metaanalyse von 13 Studien aus Asien $(n=4)$, Amerika $(n=5)$ sowie Europa $(n=4)$ beruhigen: Demnach gibt es keine Korrelation zwischen Eierverzehr und Krebsrisiko [Li F et al. Nutr Cancer 2013; 65: 538-46]. Dies gilt allerdings nicht für jeden.

Für Bewohner Kanadas, der USA und Uruguay hingegen machte es durchaus einen Unterschied, wie viele Eier sie verzehrt hatten: Verglichen mit denjenigen, die auf Eier weitgehend verzichtet hatten (niedrigste Quartile), lag das Blasenkrebsrisiko bei ausgesprochenen Eierliebhabern (höchste Quartile) um $40 \%$ höher. Entscheidend war dabei die Zubereitungsart: Ungünstig aufs Blasenkrebsrisiko wirkten sich vor allem gebratene Eier aus. Bei Asiaten und Europäern hingegen änderte die verzehrte Eiermenge am Risiko nichts.

Dass die Zubereitungsart einen Unterschied im Hinblick auf das Blasenkrebsrisiko macht, erklären sich die Studienautoren folgendermaßen: Beim Braten der Eier entstehe aufgrund der hohen Temperatur karzinogene heterozyklische Amine. Außerdem steige mit dem Bratfett die Cholesterinmenge, die aufgenommen wird, die bekanntermaßen das Blasenkrebsrisiko beeinflusse.

Dr. Dagmar Kraus 